\title{
POSTULATION OF DISJOINT UNIONS OF LINES AND A MULTIPLE POINT, II
}

\author{
E. BALLICO
}

\begin{abstract}
We study the postulation of a general union $X \subset \mathbb{P}^{3}$ of one mpoint $m P$ and $t$ disjoint lines. We prove that it has the expected Hilbert function, proving a conjecture by E. Carlini, M. V. Catalisano and A. V. Geramita.
\end{abstract}

\section{INTRODUCTION}

A scheme $X \subset \mathbb{P}^{r}$ is said to have maximal rank if for all integers $t>0$ the restriction map $H^{0}\left(\mathcal{O}_{\mathbb{P}^{r}}(t)\right) \rightarrow H^{0}\left(X, \mathcal{O}_{X}(t)\right)$ is either injective or surjective, i.e. if either $h^{0}\left(\mathcal{I}_{X}(t)\right)=0$ or $h^{1}\left(\mathcal{I}_{X}(t)\right)=0$, i.e. if $X$ imposes the " expected " number of conditions to the vector space of all homogeneous degree $t$ polynomials in $r+1$ variables. R. Hartshorne and A. Hirschowitz proved that for all integers $t>0$ and $r \geq 3$ a general union $X \subset \mathbb{P}^{r}$ of $t$ general lines has maximal rank. E. Carlini, M. V. Catalisano and A. V. Geramita considered several cases in which we allow unions of linear spaces with certain multiplicities [2], 3], 4]). We recall that for each $P \in \mathbb{P}^{r}$ the m-point $m P$ of $\mathbb{P}^{r}$ is the closed subscheme of $\mathbb{P}^{r}$ with $\left(\mathcal{I}_{P}\right)^{m}$ as its ideal sheaf. E. Carlini, M. V. Catalisano and A. V. Geramita proved that for all $r \geq 4, m>0$ and $d>0$ a general union of an m-point and $d$ disjoint lines has maximal rank (4]). In the case $r=3$ they proved that there are some exceptional cases (the one with $2 \leq d \leq m$ and $t=m$ ); in 4 the failure of maximal rank for these cases is exactly described, i.e. all positive integers $h^{0}\left(\mathcal{I}_{X}(t)\right)$ and $h^{1}\left(\mathcal{I}_{X}(t)\right)$ are computed ([4, Theorem 4.2, part (ii)]). They conjectured in [4 that these are the only exceptional cases and proved the conjecture in some cases (e.g. if $m=2$ by [4, Theorem 4.2, part (i)(e)]). In [1 their conjecture was proved when $m=3$ and an asymptotic result was proved for arbitrary $m$ ([1, Propositions 1 and 2]). In this paper we prove their conjecture in the case $m=3$, i.e. we prove the following result.

Theorem 1. Fix integers $m \geq 2, t>0$ and $d>0$. If $2 \leq d \leq m$, then assume $t \geq m+1$. Let $Y \subset \mathbb{P}^{3}$ be a general union of d lines. Then either $h^{1}\left(\mathcal{I}_{m P \cup Y}(t)\right)=0$ or $h^{0}\left(\mathcal{I}_{m P \cup Y}(t)\right)=0$.

A crucial step of the proof is contained in [4, Theorem 4.2, part (i)(c)]: the proof of the case $d=m+2$ and $t=m+1$. Let $Y \subset \mathbb{P}^{3}$ be a general union of $m+2$ lines. They proved that $h^{i}\left(\mathcal{I}_{m P \cup Y}(m+1)\right)=0, i=0,1$. After 7 and [6] it is well-known that if certain crucial curves or unions of curves and points, say $X_{1}$ and

2010 Mathematics Subject Classification. 14N05; 14H99.

Key words and phrases. postulation; Hilbert function; fat point; unions of lines; sundials; reducible conics.

The author was partially supported by MIUR and GNSAGA of INdAM (Italy). 
$X_{2}$, have $h^{i}\left(\mathcal{I}_{X_{1}}\left(t_{0}\right)\right)=0, i=0,1$, and $h^{i}\left(\mathcal{I}_{X_{2}}\left(t_{0}+1\right)\right)=0, i=0,1$, then it should be easy to control the postulation of all curves of degree $\geq \operatorname{deg}\left(X_{2}\right)$ with respect to all forms of degree $\geq t_{0}+2$. In our case by [4, Theorem 4.2, part (i)(c)] we may take $X_{1}=m P \cup Y$ with $\operatorname{deg}(Y)=m+2$. The key part of the proof is the construction of a good $X_{2}$ for $t_{0}+1=m+2$ and then to control the cases $t=t_{0}+3$ and $t=t_{0}+4$.

We work over an algebraically closed field $\mathbb{K}$. As far as we understand none of our quotations of [4] require the characteristic zero assumption made in [4].

\section{Preliminaries}

For any integer $d>0$ let $L(d)$ be the set of all unions $Y \subset \mathbb{P}^{3}$ of $d$ disjoint lines. For any $P \in \mathbb{P}^{3}$ set $L(P, d):=\{Y \in L(d): P \notin Y\}$. If $P$ is a smooth point of a scheme $T$ let $\{m P, T\}$ be the closed subscheme of $T$ with $\left(\mathcal{I}_{P, T}\right)^{m}$ as its ideal sheaf. We write $m P$ instead of $\left\{m P, \mathbb{P}^{3}\right\}$. For any positive-dimensional $A \subseteq \mathbb{P}^{3}$ and any smooth point $O$ of $A$ a tangent vector of $A$ with $O$ as its support is a degree 2 connected zero-dimensional scheme $v \subset A$ such that $\operatorname{deg}(v)=2$ and $v_{\text {red }}=\{O\}$.

Let $F \subset \mathbb{P}^{3}$ be any surface. Set $t:=\operatorname{deg}(F)$. For each closed subscheme $Z \subset \mathbb{P}^{3}$ let $\operatorname{Res}_{F}(Z)$ denote the residual scheme of $Z$ with respect to $F$, i.e. the closed subscheme of $\mathbb{P}^{3}$ with $\mathcal{I}_{Z}: \mathcal{I}_{F}$ as its ideal sheaf. If $Z$ is reduced, then $\operatorname{Res}_{F}(Z)$ is the union of the irreducible components of $Z$ not contained in $F$. Now assume $Z=m P$ for some $m>0$ and some $P \in \mathbb{P}^{3}$. If $P \notin F$, then $\operatorname{Res}_{F}(m P)=m P$. If $P$ is a smooth point of $F$, then $\operatorname{Res}_{F}(m P)=(m-1) P$ (with the convention $0 P=\emptyset$ ). For any integer $x \geq t$ we have an exact sequence

$$
0 \rightarrow \mathcal{I}_{\operatorname{Res}_{F}(Z)}(x-t) \rightarrow \mathcal{I}_{Z}(x) \rightarrow \mathcal{I}_{Z \cap F, F}(x) \rightarrow 0
$$

Hence

$$
\begin{aligned}
& \text { - } h^{0}\left(\mathcal{I}_{Z}(x)\right) \leq h^{0}\left(\mathcal{I}_{\operatorname{Res}_{F}(Z)}(x-t)\right)+h^{0}\left(F, \mathcal{I}_{Z \cap F}(x)\right) ; \\
& \text { - } h^{1}\left(\mathcal{I}_{Z}(x)\right) \leq h^{1}\left(\mathcal{I}_{\operatorname{Res}_{F}(Z)}(x-t)\right)+h^{1}\left(F, \mathcal{I}_{Z \cap F}(x)\right) .
\end{aligned}
$$

As in [2], 3, Lemma 3.3] and [4] we will call "the Castelnuovo's inequality" any of these two inequalities. If $F$ is either a plane or a smooth quadric, $D$ is an effective divisor of $F$ and $Z \subset F$ is a closed subscheme of $F$, $\operatorname{Res}_{D}(Z)$ is the closed subscheme of $F$ with $\mathcal{I}_{Z, F}: \mathcal{I}_{D, F}$ as its ideal sheaf (of course, $\mathcal{I}_{D, F} \cong \mathcal{O}_{F}(-D)$ as abstract line bundles on $F$ ). We also have the corresponding Castelnuovo's exact sequence of $\operatorname{Res}_{D}$ and the associated Castelnuovo's inequalities.

Set $0 P:=\emptyset$. We use the convention that $\left(\begin{array}{l}t \\ 3\end{array}\right)=0$ if $-2 \leq t \leq 2$ and $\left(\begin{array}{c}t+2 \\ 2\end{array}\right)=0$ if $-1 \leq t \leq 1$. We have $\operatorname{deg}(0 P)=0=\left(\begin{array}{l}2 \\ 3\end{array}\right)$. For all integers $m \geq 0$ and $k \geq 0$ define the integers $a_{m, k}$ and $b_{m, k}$ by the relations

$$
\left(\begin{array}{c}
m+1 \\
3
\end{array}\right)+(k+1) a_{m, k}+b_{m, k}=\left(\begin{array}{c}
k+3 \\
3
\end{array}\right), 0 \leq b_{m, k} \leq k
$$

If $k \geq 2$ from (1) for $k, k-2, k-1$ and $m-1$ we get

$$
2 a_{m, k-2}+(k+1)\left(a_{m, k}-a_{m, k-2}\right)+b_{m, k}-b_{m, k+1}=(k+1)^{2}
$$

Taking the difference of (2) with $k=m+2$ and the same equation with $\left(m^{\prime}, k^{\prime}\right)=$ $(m-1, m+1)$ and using that $\left(\begin{array}{c}m+2 \\ 3\end{array}\right)-\left(\begin{array}{c}m+1 \\ 3\end{array}\right)=\left(\begin{array}{c}m+1 \\ 2\end{array}\right)$ and $\left(\begin{array}{c}m+4 \\ 2\end{array}\right)-\left(\begin{array}{c}m+1 \\ 2\end{array}\right)=3 m+6$, we get

$$
a_{m-1, m+1}+(m+3)\left(a_{m, m+2}-a_{m-1, m+2}\right)+b_{m, m+2}-b_{m-1, m+1}=3 m+6
$$


for all $m>0$. Taking $k=m+2$ in (1) we get

$$
(m+3) a_{m, m+2}+b_{m, m+2}=\left(3 m^{2}+15 m+30\right) / 2
$$

Remark 1. We have $b_{m, m+1}=0$ and $a_{m, m+1}=m+2$ for all $m$. From (4) we get that if $m$ is even, then $a_{m, m+2}=3 m / 2+3$ and $b_{m, m+2}=1$, while if $m$ is odd, then $a_{m, m+2}=3 m / 2+5 / 2$ and $b_{m, m+2}=m / 2+5 / 2$. Hence for all $m \geq 3$ we have $a_{m-1, m+1}>m, a_{m, m+2}=a_{m-1, m+1}+2$ if $m$ is even and $a_{m, m+2}=a_{m-1, m+1}+1$ if $m$ is odd. We have $\left(\begin{array}{c}m+6 \\ 3\end{array}\right)-\left(\begin{array}{c}m+2 \\ 3\end{array}\right)=2 m^{2}+12 m+10$ and hence $a_{m, m+3}=2 m+4$ and $b_{m, m+3}=4$ for all $m \geq 1, a_{0,3}=6, b_{0,3}=2$. We have $\left(\begin{array}{c}m+7 \\ 3\end{array}\right)-\left(\begin{array}{c}m+2 \\ 3\end{array}\right)=$ $\left(5 m^{2}+35 m+70\right) / 2$. If $m$ is even and $m \geq 6$, then $a_{m, m+4}=5 m / 2+5$ and $b_{m, m+4}=10$. If $m \in\{2,4\}$, then $a_{m, m+4}=5 m / 2+6$ and $b_{m, m+4}=5-m$. If $m$ is odd and $m \geq 17$, then $a_{m, m+4}=5 m / 2+9 / 2$ and $b_{m, m+4}=(m+25) / 2$. If $m \in\{3,5,7,9,11,13,15\}$, then $a_{m, m+4}=5 m / 2+11 / 2$ and $b_{m, m+4}=(15-m) / 2$.

For all positive integers $m, d$ the critical value of the pair $(m, d)$ is the minimal integer $k \geq m$ such that $\left(\begin{array}{c}m+2 \\ 3\end{array}\right)+(k+1) d \leq\left(\begin{array}{c}k+3 \\ 3\end{array}\right)$. Let $W \subset \mathbb{P}^{3}$ be a union of $d$ disjoint lines with $P \notin W$. The scheme $m P \cup W$ has maximal rank if and only if $h^{0}\left(\mathcal{I}_{m P \cup W}(k-1)\right)=0$ and $h^{1}\left(\mathcal{I}_{m P \cup W}(k)\right)=0$, where $k$ is the critical value of $(m, d)$. Using (2) it is easy to check that for a fixed integer $m>0$ the sequence $a_{m, k}$ is strictly increasing for all $k \geq m-1$ (we have $a_{m, m-1}=0$ ). The integer $k$ is the critical value of the pair $(m, d)$ if and only if $a_{m, k-1}<d \leq a_{m, k}$.

\section{Assertions $B(m), R(m)$ And $H_{m, k}$}

For every odd positive integer $m$ we define Assertion $B(m)$ in the following way.

Assertion $B(m), m \geq 1, m$ odd: There is a 7 -ple $(Y, L, R, S, O, H, v)$ with the following properties:

(1) $H$ is a plane containing $P, L$ and $R$ are lines of $H, L \neq R, P \notin L \cup R$, and $\{O\}:=L \cap R$

(2) $Y$ is a union of $a_{m, m+2}$ disjoint lines, $P \notin Y$ and $Y \cap H$ is finite;

(3) $S \subset H \cap Y, \sharp(S)=b_{m, m+2}-2$;

(4) $v$ is a disjoint union of $b_{m, m+2}-2$ tangent vectors of $\mathbb{P}^{3}$, each of them with a point of $S$ as its support;

(5) $\sharp(S \cap L)=\lceil(m+3) / 4\rceil, \sharp(S \cap R)=\lfloor(m+3) / 4\rfloor$ and $L \cap R \cap S=\emptyset$;

(6) $h^{1}\left(\mathcal{I}_{m P \cup Y \cup v \cup\{O\}}(m+2)\right)=0$.

Take $(Y, L, R, S, O, H, v)$ satisfying the second, third and fourth of the conditions of $B(m)$. We have $h^{0}\left(\mathcal{O}_{m P \cup Y \cup v \cup\{O\}}(m+2)\right)=\left(\begin{array}{c}m+5 \\ 3\end{array}\right)-1$ and hence $h^{1}\left(\mathcal{I}_{m P \cup Y \cup v \cup\{O\}}(m+2)\right)=h^{0}\left(\mathcal{I}_{m P \cup Y \cup v \cup\{O\}}(m+2)\right)-1$.

For every even integer $m \geq 2$ we define Assertion $B(m)$ in the following way.

Assertion $B(m), m \geq 2, m$ even: There is a quadruple $(Y, L, R, H)$ with the following properties:

(1) $H$ is a plane containing $P, L$ and $R$ are lines of $H, L \neq R$, and $P \notin L \cup R$;

(2) $Y$ is a union of $a_{m, m+2}$ disjoint lines, $P \notin Y$ and $Y \cap H$ is finite;

(3) $\sharp((Y \cap H) \cap L)=\lceil(m+2) / 4\rceil, \sharp((Y \cap H) \cap R)=\lfloor(m+2) / 4\rfloor$;

(4) $h^{1}\left(\mathcal{I}_{m P \cup Y}(m+2)\right)=0$.

The last condition of $B(m), m$ even, is equivalent to $h^{0}\left(\mathcal{I}_{m P \cup Y}(m+2)\right)=1$.

Lemma 1. $B(m)$ is true for all $m \geq 2$. 
Proof. We first prove $B(2)$. Let $Y \subset \mathbb{P}^{3}$ be a general union of 6 lines (hence $P \notin Y$ ). By [4, part (i)(e) of Theorem 4.2] we have $h^{1}\left(\mathcal{I}_{2 P \cup Y}(4)\right)=0$. Let $H \subset \mathbb{P}^{3}$ be a general plane though $P$. Moving $Y$ we see that we may assume that no 3 of the points of $(Y \cap H) \cup\{P\}$ are collinear.

Now assume $m \geq 3$ and that $B(m-1)$ is true.

(a) In this step we assume that $m$ is odd. Take $(Y, L, R, S, H)$ satisfying $B(m-1)$. We have $h^{1}\left(\mathcal{I}_{(m-1) P \cup Y}(m+1)\right)=1$. Let $D \subset H$ be a general line. Let $v \subset H$ be a union of tangent vectors of $H$ with $S$ as its support, but no tangent vector being a tangent vector of $L \cup R$. We first check that $h^{1}\left(\mathcal{I}_{m P \cup Y \cup D \cup v \cup\{O\}}(m+\right.$ $2))=0$. Since $\operatorname{Res}_{H}(m P \cup Y \cup D \cup v \cup\{O\})=(m-1) P \cup Y$ and $h^{1}\left(\mathcal{I}_{(m-1) P \cup Y}(m+\right.$ $1))=0$, it is sufficient to prove that $h^{1}\left(H, \mathcal{I}_{((m P \cup Y) \cap H) \cup D \cup v \cup\{O\}}(m+2)\right)=0$, i.e. $h^{1}\left(H, \mathcal{I}_{((m P \cup Y) \cap H) \cup v \cup\{O\}}(m+1)\right)=0$. The scheme $((m P \cup Y) \cap H) \cup v \cup\{O\}$ is a general union of $\{m P, H\}$, the scheme $v \cup\{O\}$ and $a_{m-1, m+1}-(m+1) / 2$ general points of $H$. Hence it has degree $\left(\begin{array}{c}m+1 \\ 2\end{array}\right)+2(m+1) / 2+3(m-1) / 2+3-(m+1) / 2+1=$ $\left(\begin{array}{c}m+3 \\ 2\end{array}\right)$. We deform $D$ in a flat family of lines outside $H$ (we may do it even fixing either the point of $D \cap L$ or the point of $D \cap R$ ). For general $v$ it is easy to check that $h^{1}\left(H, \mathcal{I}_{\{m P, H\} \cup v \cup\{O\}}(m+1)\right)=0$ (order the points of $S$ and then add the corresponding connected component $v_{i}$ of $v$ following the ordering first with the point $P_{i}$ of $S$ general in a component of $L \cup R$ and then with $v_{i}$ general among the tangent vectors of $H$ with $P_{i}$ as its support; at each point use that $h^{0}\left(H, \mathcal{O}_{\{m P, H\}}(m)\right)=m+1$ and that if $P_{i} \in L_{i}$, then $\left|\mathcal{I}_{\{m P, H\} \cup\left\{2 P_{i}, L_{i}\right\}}(m+1)\right| \cong$ $\left.\left|\mathcal{I}_{\{m P, H\}}(m)\right|\right)$. Since $Y \cap H \backslash S$ is general in $H$, we get $h^{i}\left(H, \mathcal{I}_{((m P \cup Y) \cap H) \cup v \cup\{O\}}(m+\right.$ 1)) $=0, i=0,1$.

(b) In this step we assume that $m$ is even. Take $(Y, L, R, S, O, H, v)$ satisfying $B(m-1)$. Let $w \subset \mathbb{P}^{3}$ be a general tangent vector with $O$ as its support. The scheme $Y \cup L \cup R \cup w \cup v$ is a flat limit of a family of disjoint unions of $a_{m, m+2}$ lines (i.e. there are a flat family $\left\{Y_{t}\right\}_{t \in \Gamma}, \Gamma$ an integral affine curve, $o \in \Gamma, Y_{o}=Y \cup L \cup R \cup w \cup v$ ) such that $Y \subset Y_{t}$, say $Y_{t}=Y \cup L_{t} \cup R_{t}$ for all $t$ with $\left\{L_{t}\right\}$, and $\left\{R_{t}\right\}$ flat families with $L_{o}=L$ and $L_{o}=L$, and either $L_{t} \cap L \neq \emptyset$ for all $t, R_{t} \cap R=\emptyset$ for all $t \neq o$ (case $m \equiv 2(\bmod 4))$ or $R_{t} \cap R \neq \emptyset$ for all $t$ and $L_{t} \cap L=\emptyset$ for all $t \neq o$ (case $m \equiv 0$ $(\bmod 4))$. We may take as the new set $S$ the set $S \cup\left(L_{t} \cup R_{t}\right) \cap(L \cup R)$ for a general $t \in \Gamma$. By the semicontinuity theorem for cohomology ([5, III.12.8]) it is sufficient to prove that $h^{1}\left(\mathcal{I}_{m P \cup Y \cup L \cup R \cup v \cup w}(m+2)\right)=0$. Since $(m P \cup Y \cup L \cup R \cup v \cup w) \cap H=$ $\{m P, H\} \cup L \cup R \cup((Y \cap H) \backslash S)$ and $\operatorname{Res}_{H}(m P \cup Y \cup L \cup R \cup v \cup w)=(m-1) P \cup Y \cup$ $v \cup\{O\}$, it is sufficient to prove that $h^{1}\left(H, \mathcal{I}_{\{m P, H\} \cup L \cup R \cup((Y \cap H) \backslash S)}(m+2)\right)=0$, i.e. $h^{1}\left(\left(H, \mathcal{I}_{\{m P, H\} \cup((Y \cap H) \backslash S)}(m)\right)=0\right.$. This is true, because $(Y \cap H) \backslash S$ is general in $H$ and $\sharp((Y \cap H) \backslash S)=3 m / 2+1-m / 2=m+1=h^{0}\left(H, \mathcal{I}_{\{m P, H\}}(m)\right.$.

Remark 2. Fix $(m, d)$ with critical value $m+1$ and degree $d \geq m+1$. Let $W \subset \mathbb{P}^{m}$ be a general union of $d$ lines. Since $a_{m, m+1}=m+2$, we have $m+1 \leq d \leq m+2$. By [4, part (i)(c) of Theorem 4.2] the scheme $m P \cup W$ has maximal rank.

Lemma 2. Fix an integer $d \leq a_{m, m+2}$ and let $X \subset \mathbb{P}^{3}$ be a general union of $d$ lines. Then $h^{1}\left(\mathcal{I}_{m P \cup X}(m+2)\right)=0$.

Proof. This statement is obvious if $m=1$ by [6]. Assume $m \geq 2$. It is sufficient to find a disjoint union $W$ of $d$ lines such that $P \notin W$ and $h^{1}\left(\overline{\mathcal{I}}_{m P \cup W}(m+2)\right)=0$. Take a solution of $B(m)$ and call $Y$ the curve in it. Take as $W$ the union of $d$ of the lines of $Y$. 
For all odd integer $m \geq 3$ let $R(m)$ denote the following assertion:

Assertion $R(m), m$ odd, $r \geq 3$ : There exists a quintuple $(Y, S, D, H, v)$ with the following properties:

(1) $Y \subset \mathbb{P}^{3}$ is a disjoint union of $3 m / 2+5 / 2$ lines, $P \notin Y, H$ is a plane containing $P, D \subset H$ is a smooth conic such that $P \notin D$ and $S:=(Y \cap$ $H) \cap D$ has cardinality $m / 2+5 / 2$;

(2) $v \subset \mathbb{P}^{3}$ is a disjoint union of tangent vectors of $\mathbb{P}^{3}$ with $v_{\text {red }}=S$; no connected component of $v$ is contained in $Y$;

(3) $h^{i}\left(\mathcal{I}_{m P \cup Y \cup v}(m+2)\right)=0$.

Lemma 3. $R(m)$ is true for all odd integers $m \geq 3$.

Proof. Take $(Y, L, R, H)$ satisfying $B(m-1)$. We have $\sharp(Y \cap(L \cup R))=(m+1) / 2$, $h^{1}\left(\mathcal{I}_{(m-1) P \cup Y}(m+1)\right)=0$ and $h^{0}\left(\mathcal{I}_{(m-1) P \cup Y}(m+1)\right)=1$. Since $h^{0}\left(\mathcal{I}_{(m-2) P \cup Y}(m)\right)=$ $0, P \in H$, and $Y \cap(R \cup L) \neq Y \cap H$, there is $o \in L \cup R$ not in the base locus of $\left|\mathcal{I}_{(m-1) P \cup Y}(m+1)\right|$ and hence $h^{i}\left(\mathcal{I}_{(m-1) P \cup Y \cup\{o\}}(m+1)\right)=0, i=0,1$. We may deform $(Y, L \cup R, o)$ to $\left(Y^{\prime}, C, o^{\prime}\right)$, where $C \subset H$ is a smooth conic, $P \notin C$, $o^{\prime} \in C \backslash C \cap Y, \sharp\left(Y^{\prime} \cap C\right)=(m+1) / 2$ and $h^{0}\left(\mathcal{I}_{(m-1) P \cup Y \cup\left\{o^{\prime}\right\}}(m+1)\right)=0$. We may take as $o^{\prime}$ a general point of $C$. Let $o^{\prime \prime}$ be another general point of $C$ and call $T$ the line spanned by $o^{\prime}$ and $o^{\prime \prime}$ (alternatively, take a general line $T \subset H$ and set $\left.\left\{o^{\prime}, o^{\prime \prime}\right\}:=C \cap T\right)$. Let $w \subset H$ be a general union of tangent vectors of $H$, each of them supported by a different point of $Y \cap(L \cup R)$. Let $v^{\prime} \subset \mathbb{P}^{3}$ be a general tangent vector of $\mathbb{P}^{3}$ with $o^{\prime}$ as its support (hence $\left.\operatorname{Res}_{H}\left(v^{\prime}\right)=\left\{o^{\prime}\right\}\right)$. Let $v^{\prime \prime} \subset H$ be a general tangent vector of $H$ with $v_{\text {red }}^{\prime \prime}=\left\{o^{\prime \prime}\right\}$. Since $v^{\prime \prime} \subset H$, we have $\operatorname{Res}_{H}\left(v^{\prime \prime}\right)=\emptyset$. Since $v^{\prime \prime}$ is general, it is not tangent to $T$ and hence $\operatorname{Res}_{T}\left(v^{\prime \prime}\right)=\left\{o^{\prime \prime}\right\}$. Take $Y^{\prime}:=Y \cup T, v:=w \cup v^{\prime} \cup v^{\prime \prime}$ and $S:=Y \cap(L \cup R) \cup\left\{o^{\prime}, o^{\prime \prime}\right\}$. We want to check that the quintuple $\left(Y^{\prime}, S, C, H, v\right)$ satisfies $R(m)$. The scheme $v$ is a union of tangent vectors, one for each point of $S$. We have $\sharp(S)=(m+1) / 2+2=(m+5) / 2$. The set $S$ is contained in the smooth conic $D$. It is sufficient to check that $h^{i}\left(\mathcal{I}_{m P \cup Y^{\prime} \cup v}(m+2)\right)=0, i=0,1$. Since $\operatorname{Res}_{H}\left(m P \cup Y^{\prime} \cup v\right)=(m-1) P \cup Y \cup\left\{o^{\prime}\right\}, h^{i}\left(\mathcal{I}_{(m-1) P \cup Y \cup\{o\}}(m+1)\right)=0, i=0,1$, $o^{\prime} \in D,\left(m P \cup Y^{\prime} \cup v\right) \cap H=\left\{(m-1) P \cup w \cup T \cup\left\{o^{\prime}\right\} \cup v^{\prime \prime} \cup((Y \cap H) \backslash(Y \cap H) \cap(L \cup R))\right.$ and $\operatorname{Res}_{T}\left(\{m P, H\} \cup T \cup v^{\prime \prime} \cup(Y \cap H) \cup w\right)=\{m P, H\} \cup(Y \cap H) \cap w \cup\left\{o^{\prime \prime}\right\}$, it is sufficient to prove that $h^{i}\left(H, \mathcal{I}_{\{m P, H\} \cup(Y \cap H) \cup w \cup\left\{o^{\prime \prime}\right\}}(m+1)\right)=0$. We have $\operatorname{deg}\left(\{m P, H\} \cup(Y \cap H) \cup w \cup\left\{o^{\prime \prime}\right\}\right)=\left(\begin{array}{c}m+1 \\ 2\end{array}\right)+3 m / 2+3 / 2+(m+1) / 2+1=\left(\begin{array}{c}m+3 \\ 2\end{array}\right)$. Use again that $h^{1}\left(H, \mathcal{I}_{\{m P, H\} \cup w}(m+1)\right)^{2}=0$ (as in part (a) of the proof of Lemma 11) and that $Y \cap H \backslash v_{\text {red }}$ is general in $H$.

Lemma 4. Fix an integer $d>a_{m, m+2}$ and let $X \subset \mathbb{P}^{3}$ be a general union of $d$ lines. Then $h^{0}\left(\mathcal{I}_{m P \cup X}(m+2)\right)=0$.

Proof. It is sufficient to prove the lemma when $d=a_{m, m+2}+1$. First assume that $m$ is even. Take a solution $(Y, L, R, H)$ of $B(m)$. Since $h^{0}\left(\mathcal{I}_{m P \cup Y}(m+2)\right)=1$, we have $h^{0}\left(\mathcal{I}_{m P \cup Y \cup D}(m+2)\right)=0$ for any line $D$ through a general point of $\mathbb{P}^{3}$.

Now assume that $m$ is odd. Let $W \subset \mathbb{P}^{3}$ be a general union of $a_{m-1, m+1}$ lines. Since $B(m-1)$ is true, we have $h^{1}\left(\mathcal{I}_{(m-1) P \cup W}(m+1)\right)=0$ and hence $h^{0}\left(\mathcal{I}_{(m-1) P \cup W \cup o}(m+1)\right)=0$ for a general $o \in \mathbb{P}^{3}$. Let $M \subset \mathbb{P}^{3}$ be a general plane containing $\{P, o\}$. Let $L^{\prime}, R^{\prime} \subset M$ be two general lines through $o$. It is sufficient to prove that $h^{0}\left(\mathcal{I}_{m P \cup W \cup L^{\prime} \cup R^{\prime} \cup 2 o}(m+2)\right)=0$. Since $\operatorname{Res}_{M}\left(m P \cup W \cup L^{\prime} \cup R^{\prime} \cup 2 o\right)=$ $(m-1) P \cup W \cup\{o\}$, it is sufficient to prove that $h^{0}\left(H, \mathcal{I}_{\{m P, H\} \cup(W \cap H) \cup L^{\prime} \cup R^{\prime}}(m+\right.$ 
$2))=0$, i.e. $h^{0}\left(H, \mathcal{I}_{\{m P, H\} \cup(W \cap H)}(m)\right)=0$. Since $W \cap H$ is a general union of $a_{m-1, m+1}>m$ points of $H$, we have $h^{0}\left(H, \mathcal{I}_{\{m P, H\} \cup(W \cap H)}(m)\right)=0$.

Consider the following statement:

Assertion $H_{m, k}, m>0, k \geq m+2$ : There exist a quintuple $(Y, Q, S, v, E)$ with the following properties:

(1) $Y \in L\left(P, a_{m, k}\right), Q$ is a smooth quadric surface intersecting transversally $Y$, $P \notin Q$

(2) $S \subseteq Y \cap Q, \sharp(S)=b_{m, k}$ and $v \subset \mathbb{P}^{3}$ is a disjoint union of tangent vectors with $v_{\text {red }}=S$ and no connected component of $v$ contained in $Y$;

(3) $E \subset Q$ is a disjoint union of $\left\lceil b_{m, k} / 2\right\rceil$ lines, $S \subset E$ and each component of $E$ contains at most two points;

(4) $h^{i}\left(\mathcal{I}_{m P \cup Y \cup v}(k)\right)=0, i=0,1$.

Take $(Y, Q, S, v, E)$ satisfying the first two conditions of the definition of $H_{m, k}$. We have $h^{0}\left(\mathcal{O}_{m P \cup Y \cup v}(k)\right)=\left(\begin{array}{c}k+3 \\ 3\end{array}\right)$ and hence $h^{0}\left(\mathcal{I}_{m P \cup Y \cup v}(k)\right)=h^{1}\left(\mathcal{I}_{m P \cup Y \cup v}(k)\right)$. Now assume that $(Y, Q, S, v, E)$ satisfies the third condition of the definition of $H_{m, k}$. If $b_{m, k}$ is even, then each line of $S$ contains exactly two points of $S$. If $b_{m, k}$ is odd, then $\sharp(S \cap L)=2$ for $\left(b_{m, k}-1\right) / 2$ of the components of $E$, while $\sharp(S \cap L)=1$ for the other component.

From now on $Q \subset \mathbb{P}^{3}$ is a smooth quadric surface such that $P \notin Q$.

Lemma 5. $H_{m, m+3}$ is true for all $m>0$.

Proof. We have $a_{m, m+1}=m+2, b_{m, m+1}=0, a_{m, m+3}=2 m+4$ and $b_{m, m+3}=4$ (Remark 11). Let $Y \subset \mathbb{P}^{3}$ be a general union of $m+2$ lines. By [4, Part (i)(c) of Theorem 4.2] we have $h^{i}\left(\mathcal{I}_{Y}(m+1)\right)=0, i=0,1$. For a general $Y$ we may assume that $Y \cap Q$ is formed by $2 m+4$ general points of $Q$. Let $F \subset Q$ be a general union of $m+2$ lines of type $(0,1)$. Fix $S_{1} \subset Y \cap Q$ such that $\sharp\left(S_{1}\right)=2$. Let $E^{\prime} \subset Q$ be the union of the lines of type $(1,0)$ containing a point of $S_{1}$. Fix $S_{2} \subset E^{\prime} \cap F$ such that $\sharp\left(S_{2} \cap L\right)=1$ for each component $L$ of $E^{\prime}$ and that no component of $F$ contains two points of $S_{2}$. Set $S:=S_{1} \cup S_{2}$ and call $v \subset Q$ a general union of tangent vectors of $Q$ with $S$ as its support. We claim that $h^{i}\left(\mathcal{I}_{m P \cup Y \cup F \cup v}(m+3)\right)=0$. Since $\operatorname{Res}_{Q}(m P \cup Y \cup F \cup v)=m P \cup Y, Q \cap(m P \cup Y \cup F \cup v)=F \cup v \cup((Y \cap$ $\left.Q) \backslash S_{1}\right), \operatorname{Res}_{F}\left(F \cup v \cup\left((Y \cap Q) \backslash S_{1}\right)=\left((Y \cap Q) \backslash S_{1}\right) \cup v_{\text {red }}=Y \cap Q \cup S_{2}\right.$ and $\left.h^{i}\left(\mathcal{I}_{m P \cup Y}(m+1)\right)\right)=0, i=0,1$, to prove the claim it is sufficient to prove that $h^{i}\left(Q, \mathcal{I}_{\left((Y \cap Q) \backslash S_{1}\right) \cup v}(m+3,1)\right)=0, i=0,1$. We have $\operatorname{deg}\left(\left((Y \cap Q) \backslash S_{1}\right) \cup S\right) 2 m+6$. Hence it is sufficient to use that $h^{1}\left(Q, \mathcal{I}_{S}(m+3,1)\right)=0(S$ is the union of two degree 2 schemes on two different lines of type $(1,0))$ and $(Y \cap Q) \backslash S_{1}$ is general in $Q$.

Lemma 6. $H_{m, m+4}$ is true for all $m \geq 2$.

Proof. The proof depends on the parity of $m$.

(a) First assume that $m$ is even. We have $a_{m, m+2}=3 m / 2+3$ and $b_{m, m+2}=1$.

(a1) Assume for the moment $m \geq 6$ and hence $a_{m, m+4}=5 \mathrm{~m} / 2+5$ and $b_{m, m+4}=10$ (Remark 1). Let $Y \subset \mathbb{P}^{3}$ be a general union of $a_{m, m+2}=3 m / 2+3$ lines. Since $B(m)$ is true (Lemma11) and $b_{m, m+2}=1$, we have $h^{1}\left(\mathcal{I}_{m P \cup Y}(m+2)\right)=$ 0 and $h^{0}\left(\mathcal{I}_{m P \cup Y}(m+2)\right)=1$. The last equality implies $h^{0}\left(\mathcal{I}_{m P \cup Y}(m+1)\right)=0$.

Let $T \subset \mathbb{P}^{3}$ be the only surface of degree $m+2$ containing $m P \cup Y$. Fix a system $x_{0}, x_{1}, x_{2}, x_{3}$ of homogeneous coordinates and let $f\left(x_{0}, x_{1}, x_{2}, x_{3}\right)$ be a degree $m+2$ homogeneous equation of $T$. In characteristic zero we have $\partial f / \partial x_{i} \neq 0$ for at least 
one index $i$. Since $\partial f / \partial x_{i} \neq 0$ and $h^{0}\left(\mathcal{I}_{m P \cup Y}(m+1)\right)=0$, we have $\partial f / \partial x_{i} \mid m P \cup$ $Y \neq 0$, i.e. $m P \cup Y \nsubseteq \operatorname{Sing}(T)$, i.e. $Y \nsubseteq \operatorname{Sing}(T)$. In characteristic $p>0$ we need to prove the existence of $Y \in L(P, 3 m / 2+3)$ such that $h^{0}\left(\mathcal{I}_{m P \cup Y}(m+2)\right)=1$ and at least one component of $Y$ is not contained in the singular locus of the only degree $m$ hypersurface containing $m P \cup Y$. We get this using the proof that $B(m-1)$ implies $B(m)$ when $m$ is even (part (b) of the proof of Lemma 1). Take (Y,L, R, S, O, H, v) satisfying $B(m-1)$ and use that $h^{0}\left(H, \mathcal{I}_{\{m P, H\} \cup 2 L \cup 2 R}(m+2)\right)=0$.

Since $Y \nsubseteq \operatorname{Sing}(T)$, there is $O \in Y$ and a tangent vector $w$ to $\mathbb{P}^{3}$ with $w \nsubseteq T$. Hence $h^{0}\left(\mathcal{I}_{m P \cup Y \cup w}(m+2)\right)=0$ and so $h^{1}\left(\mathcal{I}_{m P \cup Y \cup w}(m+2)\right)=0$. Take as $Q$ a general quadric surface through $O$. We have $P \notin Q$ and $\operatorname{Res}_{Q}(m P \cup Y \cup w)=$ $m P \cup Y \cup w$. Moving the lines of $Y$ among the unions of $a_{m, m+2}$ disjoint lines of $\mathbb{P}^{3}$, one of them containing $O$, we may assume that $(Y \cap Q) \backslash\{O\}$ is a general union of $3 m+5$ points of $Q$. Let $F \subset Q$ be a union of $m+2$ distinct lines of type $(0,1)$ of $Q$ with $\{O\}=Y \cap F$. Fix $S_{1} \subset(Y \cap Q) \backslash\{O\}$ with $\sharp\left(S_{1}\right)=5$. Let $E \subset Q$ be the union of the 5 lines of type $(1,0)$ of $Q$ containing one point of $S_{1}$. Take $S_{2} \subset E \cap F$ such that each line of $E$ contains exactly one point of $S_{2}$ and each line of $F$ contains at most one point of $S_{2}$. Set $S:=S_{1} \cup S_{2}$. Let $v \subset Q$ be a general union of tangent vectors of $Q$ with $v_{\text {red }}=S$. As in the proof of Lemma 5 it is sufficient to prove that $h^{i}\left(\mathcal{I}_{m P \cup Y \cup w \cup F \cup v}(m+4)\right)=0$, $i=0,1$. Since $h^{i}\left(\mathcal{I}_{m P \cup Y \cup w}(m+2)\right)=0, i=0,1$, it is sufficient to prove that $h^{i}\left(Q, \mathcal{I}_{(Y \cap Q) \cup F \cup v}(m+4)\right)=0$, i.e. $h^{i}\left(Q, \mathcal{I}_{(Y \cap Q) \cup v}(m+4,2)\right)=0, i=0,1$. We have $\operatorname{deg}((Y \cap Q) \cup v)=20+(3 m+6)-10$. Since $v$ is general, $\operatorname{deg}(v)=20$ and $S$ is general with the only restriction that 5 lines of type $(1,0)$ of $Q$ contain each two points of $S$, we have $h^{1}\left(\mathcal{I}_{v}(m+4,2)\right)=0$. Since $Y \cap Q \backslash S_{1}$ is general in $Q$, we get $h^{i}\left(Q, \mathcal{I}_{(Y \cap Q) \cup v}(m+4,2)\right)=0, i=0,1$.

(a2) Now assume $m \in\{2,4\}$. We have $a_{m, m+4}=5 m / 2+6$ and $b_{m, m+4}=$ $5-m$. Now $F$ is a union of $m+3$ lines, $\sharp\left(S_{1}\right)=2-m / 2, \operatorname{deg}(E)=3-m / 2$ and $\sharp\left(S_{2}\right)=3-m / 2$.

(b) Now assume that $m$ is odd.

(b1) Assume $m \geq 17$. We have $a_{m, m+2}=3 m / 2+5 / 2$ and $b_{m, m+2}=(m+5) / 2$. Since $m \geq 17$, we have $a_{m, m+4}=5 m / 2+9 / 2$ and $b_{m, m+4}=(m+25) / 2$ (Remark 1). Take a solution $(Y, S, D, H, v)$ of $R(m)$ (Lemma 3). Let $Q \subset \mathbb{P}^{3}$ be a general quadric surface containing $D$. Since $P \notin D$, then $P \notin Q$. The quadric $Q$ is smooth and it intersects transversally $Q$. By the semicontinuity theorem for cohomology (5, III.12.8]) for general $v$ we may also assume that no connected component of $v$ is contained in $Q$, i.e. that $(m P \cup Y \cup v)=Y \cap Q$ (as schemes) and that $\operatorname{Res}_{Q}(m P \cup Y \cup v)=m P \cup Y \cup v$. We may move each component of $Y$ keeping fixed its point in $D$. Hence we may assume that $Y \cap(Q \backslash D)$ is a general subset of $Q$ with cardinality $2 a_{m, m+2}-b_{m, m+2}$. We have $a_{m, m+4}-a_{m, m+2}=m+2 \geq$ $(m+5) / 2=b_{m, m+2}$. Let $F \subset Q$ be a disjoint union of $m+2$ lines of type $(0,1)$ with the only restriction that $F \cap Y=Y \cap D$ (it exists, because $m+2 \geq b_{m, m+2}$ ). Fix $S_{1} \subseteq(Y \cap Q) \backslash S$ such that $\sharp\left(S_{1}\right)=\left\lfloor b_{m, m+4} / 2\right\rfloor$ (it exists because $2 a_{m, m+2}=$ $\left.3 m+5 \geq(m+5) / 2+m \geq b_{m, m+2}+\left\lfloor b_{m, m+4} / 2\right\rfloor\right)$. Let $E_{1}$ be the union of the lines of type $(1,0)$ of $Q$ containing one point of $S_{1}$. If $b_{m, m+4}$ is even, then set $E^{\prime}:=E$. If $b_{m, m+4}$ is odd, then let $E^{\prime}$ be the union of $E_{1}$ and a general line of type $(1,0)$ of $Q$. Let $S_{2} \subset E^{\prime} \cap E^{\prime \prime}$ be the union of one point for each component of $E^{\prime}$, with the restriction that $S_{2} \cap S_{1}=\emptyset$ and that each point of $S_{2}$ is contained in a different line of $E^{\prime \prime}$; we may find such a set $S_{2}$, because $E^{\prime \prime} \cap S_{1}=\emptyset$ and 
$\operatorname{deg}\left(E^{\prime \prime}\right)=a_{m, m+4}-a_{m, m+2}=m+2 \geq\left\lceil b_{m, m+4} / 2\right\rceil$. Let $v^{\prime} \subset Q$ be a general union of $b_{m, m+4}$ tangent vectors of $Q$ with $v_{\text {red }}^{\prime}=S^{\prime}$. Since $v^{\prime}$ is general, no connected component of $v^{\prime}$ is contained in $E^{\prime \prime}$ (hence $\operatorname{Res}_{E^{\prime \prime}}\left((Y \cap Q) \cup v^{\prime}\right)=Y \cap\left(Q \backslash E^{\prime \prime}\right) \sqcup S^{\prime}=$ $((Y \cap Q) \backslash S) \sqcup S^{\prime}$.

(b2) Assume $m \in\{3,5,7,9,11,13,15\}$. We have $a_{m, m+4}-a_{m, m+2}=m+3$ and $b_{m, m+4}=(15-m) / 2$. We make the construction of step (b1) with $\operatorname{deg}(F)=m+3$, $\sharp\left(S_{1}\right)=\lfloor(15-m) / 4\rfloor$, and $\operatorname{deg}(E)=\sharp\left(S_{2}\right)=\lceil(15-m) / 4\rceil$.

Lemma 7. For all integers $k \geq m+3$ we have $a_{m, k}-a_{m, k-2} \geq a_{0, k}-a_{0, k-2}-1 \geq$ $\lceil k / 2\rceil$.

Proof. From (2) and the same equation for $m=0$ we get

$$
\begin{aligned}
& 2 a_{m, k-2}+(k+1)\left(a_{m, k}-a_{m, k-2}\right)+b_{m, k}-b_{m-k-2}= \\
& 2 a_{0, k}+(k+1)\left(a_{0, k}-a_{0, k-2}\right)+b_{0, k}-b_{0, k-2}
\end{aligned}
$$

We have $b_{0, x}=0$ if $x \equiv 0,1(\bmod 3)$ and $b_{0, x}=(x+1) / 3$ if $x \equiv 2(\bmod 3)$. The definitions of the integers $a_{m, k-2}$ and $a_{0, k-2}$ give $a_{m, k-2} \leq a_{0, k-2}$, proving the lemma.

Lemma 8. $H_{m, k}$ is true for all $k \geq m+3$.

Proof. By Lemmas 5 and 6 we may assume $k \geq m+5$ and that $H_{m, k-2}$ is true. Fix a solution $(Y, Q, S, v, E)$ of $H_{m, k-2}$. Deforming if necessary each line of $Y$ we may assume that $(Q \cap Y) \backslash S$ is a general subset of $Q$. Taking instead of $v$ a union of general tangent vectors of $\mathbb{P}^{3}$ with the points of $S$ as their support we may assume that no connected component of $v$ is contained in $Q$. Therefore $\operatorname{Res}_{Q}(Y \cup v)=Y \cup v$ and $(Y \cup v) \cap Q=Y \cap Q$ (as schemes). Call $(0,1)$ the ruling of $Q$ containing $E$ (any ruling of $Q$ if $b_{m, k-2}=0$ and hence $\left.E=\emptyset\right)$. Lemma 7 gives $a_{m, k}-a_{m, k-2} \geq \operatorname{deg}(E)$. Let $F \subset Q$ be a general union of $a_{m, k}-a_{m, k-2}-\left\lceil b_{m, k-2} / 2\right\rceil$ lines of type $(0,1)$ of $Q$. Set $E^{\prime \prime}:=E \cup F$.

Claim 1: If $k \geq m+5$, then $2 a_{m, k-2} \geq k-2+k / 2$.

Proof of Claim 1: We have $2(k-1) a_{m, k-2}+2 b_{m, k-2}=2\left(\begin{array}{c}k+3 \\ 3\end{array}\right)-2\left(\begin{array}{c}m+2 \\ 3\end{array}\right)$ and $b_{m, k-2} \leq k-2$. Set $\psi(k, m):=2\left(\begin{array}{c}k+3 \\ 3\end{array}\right)-2\left(\begin{array}{c}m+2 \\ 3\end{array}\right)-(k-1)(k-2+k / 2)-2 k+4$. It is sufficient to prove that $\psi(k, m) \geq 0$ for all $k \geq m+5$. We have $\psi(m+5, m)=$ $(m+8)(m+7)(m+6) / 3-(m+2)(m+1) m / 3-(m+4)(3 m+11) / 2-2 m+6 \geq 0$ and $\psi(k+1, m) \geq \psi(k, m)$ for all $k \geq m+5$.

Fix $S_{1} \subseteq(Y \cap Q) \backslash S$ such that $\sharp\left(S_{1}\right)=\left\lfloor b_{m, k}\right\rfloor$ (it exists by Claim 1 and the inequalities $\left.b_{m, k-2} \leq k-2, b_{m, k} \leq k\right)$. Let $E_{1} \subset Q$ be the union of the lines of type $(1,0)$ of $Q$ containing one point of $S_{1}$. If $b_{m, k}$ is even, then set $E^{\prime}:=E$. If $b_{m, k}$ is odd, then let $E^{\prime}$ be the union of $E_{1}$ and a general line of type $(1,0)$ of $Q$. Let $S_{2} \subset$ $E^{\prime} \cap E^{\prime \prime}$ be the union of one point for each component of $E^{\prime}$, with the restriction that $S_{2} \cap S_{1}=\emptyset$ and that each point of $S_{2}$ is contained in a different line of $E^{\prime \prime}$; we may find such a set $S_{2}$, because $E^{\prime \prime} \cap S_{1}=\emptyset$ and $\operatorname{deg}\left(E^{\prime \prime}\right)=a_{m, k}-a_{m, k-2} \geq\left\lceil b_{m, k} / 2\right\rceil$ (Lemma 77). Set $S^{\prime}:=S_{1} \cup S_{2}$. Let $v^{\prime} \subset Q$ be a general union of $b_{m, k}$ tangent vectors of $Q$ with $v_{\text {red }}^{\prime}=S^{\prime}$. Since $v^{\prime}$ is general, no connected component of $v^{\prime}$ is contained in $E^{\prime \prime}$ (hence $\left.\operatorname{Res}_{E^{\prime \prime}}\left((Y \cap Q) \cup v^{\prime}\right)=Y \cap\left(Q \backslash E^{\prime \prime}\right) \sqcup S^{\prime}=((Y \cap Q) \backslash S) \sqcup S^{\prime}\right)$.

Claim 2: We claim that $h^{i}\left(\mathcal{I}_{m P \cup Y \cup v \cup E^{\prime \prime} \cup v^{\prime}}(k)\right)=0, i=0,1$.

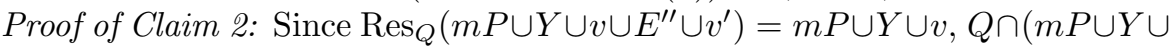
$\left.v \cup E^{\prime} \cup v^{\prime}\right)=(Y \cap Q) \cup E^{\prime \prime} \cup v^{\prime}$ and $h^{i}\left(\mathcal{I}_{m P \cup Y \cup v}(k-2)\right)=0, i=0,1$, it is sufficient to prove that $h^{i}\left(Q, \mathcal{I}_{(Y \cup Q) \cup v^{\prime} \cup E^{\prime}}(k)\right)=0, i=0,1$, i.e. $h^{i}\left(Q, \mathcal{I}_{((Y \cap Q) \backslash S) \cup S^{\prime}}(k, k-\right.$ 
$\left.\left.a_{m, k}+a_{m, k-2}\right)\right)=0, i=0,1$. By (2) we have $\left.\sharp((Y \cap Q) \backslash S) \cup S^{\prime}\right)=(k+1)(k+$ $\left.1-a_{m, k}+a_{m, k-2}\right)$. Hence it is sufficient to prove that the set $((Y \cap Q) \backslash S) \cup S^{\prime}$ gives independent conditions to the linear system $\left|\mathcal{O}_{Q}\left(k, k-a_{m, k}+a_{m, k-2}\right)\right|$. Since $(Y \cap Q) \backslash S$ is general in $Q$, it is sufficient to prove that $S^{\prime}$ gives independent conditions to $\left|\mathcal{O}_{Q}\left(k, k-a_{m, k}+a_{m, k-2}\right)\right|$. This is true since $S_{1}$ is general and hence the only restriction on the subset $S^{\prime}$ of $Q$ is that each line of $E_{1}$ contains two points of $S^{\prime}$.

We may deform $Y \cup v \cup E^{\prime \prime}$ to a family of members of $L\left(P, a_{m, k}\right)$ containing the points of $S^{\prime}$ and whose general member, $Y^{\prime}$, intersects transversally $Q$, because each line of $E^{\prime \prime}$ contains at most one point of $Q$. The quintuple $\left(Y^{\prime}, Q, S^{\prime}, v^{\prime}, E^{\prime}\right)$ satisfies $H_{m, k}$.

Proof of Theorem 1: Fix positive integers $m, d$ with critical value $k$. Hence $a_{m, k-1}<$ $d \leq a_{m, k}$. See Remark 2 and Lemma 2 for the cases $k=m, m+1, m+2$. Hence we may assume $k \geq m+3$ and that the theorem is true for the integers $d$ such that $(m, d)$ has critical value $<k$. Since $L(P, d)$ is irreducible, it is sufficient to prove the existence of $A, B \in L(P, d)$ such that $h^{0}\left(\mathcal{I}_{m P \cup B}(k-1)\right)=0$ and $h^{1}\left(\mathcal{I}_{m P \cup A}(k)\right)=0$. Let $Q \subset \mathbb{P}^{3}$ be a smooth quadric surface such that $P \notin Q$.

(a) In this step we prove the existence of $A$. Since any element of $L(P, d)$ is a union of some of the connected components of an element of $L\left(P, a_{m, k}\right)$, it is sufficient to do the case $d=a_{m, k}$. Fix a solution $(Y, Q, S, v, E)$ of $H_{m, k-2}$. Deforming if necessary each line of $Y$ we may assume that $(Q \cap Y) \backslash S$ is a general subset of $Q$. Taking instead of $v$ a union of general tangent vectors of $\mathbb{P}^{3}$ with the points of $S$ as their support we may assume that no connected component of $v$ is contained in $Q$. Therefore $\operatorname{Res}_{Q}(Y \cup v)=Y \cup v$ and $(Y \cup v) \cap Q=Y \cap Q$ (as schemes). Call $(0,1)$ the ruling of $Q$ containing $E$ (any ruling of $Q$ if $b_{m, k-2}=0$ and hence $E=\emptyset$ ). Lemma 7 gives $a_{m, k}-a_{m, k-2} \geq \operatorname{deg}(E)$. Let $F \subset Q$ be a general union of $a_{m, k}-a_{m, k-2}-\left\lceil b_{m, k-2} / 2\right\rceil$ lines of type $(0,1)$ of $Q$. The scheme $Y \cup E \cup F \cup v$ is a flat limit of a family of disjoint unions of $a_{m, k}$ lines, none of them containing $P$. By the semicontinuity theorem for cohomology to prove the existence of $A$ it is sufficient to prove that $h^{1}\left(\mathcal{I}_{m P \cup Y \cup E \cup F \cup v}(k)\right)=0$. We proved a more difficult vanishing in the proof of Lemma 8 (copy it without $v^{\prime}$ ).

(b) In this step we prove the existence of $B$. By Lemma 4 we may assume $k-1 \geq m+3$. Since $b>a_{m, k-1}$, to prove the existence of $B$ it is sufficient to prove it when $d=a_{m, k-1}+1$.

(b1) Assume for the moment $k-1 \geq m+4$, i.e. $k-3 \geq m+2$. Fix a solution $(Y, Q, S, v, E)$ of $H_{m, k-3}$. Deforming if necessary each line of $Y$ we may assume that $(Q \cap Y) \backslash S$ is a general subset of $Q$. Taking instead of $v$ a union of general tangent vectors of $\mathbb{P}^{3}$ with the points of $S$ as their support we may assume that no connected component of $v$ is contained in $Q$. Therefore $\operatorname{Res}_{Q}(Y \cup v)=Y \cup v$ and $(Y \cup v) \cap Q=Y \cap Q$ (as schemes). Call $(0,1)$ the ruling of $Q$ containing $E$ (any ruling of $Q$ if $b_{m, k-3}=0$ and hence $E=\emptyset$ ). Lemma 7 gives $a_{m, k-1}-a_{m, k-3} \geq \operatorname{deg}(E)$. Let $F \subset Q$ be a general union of $a_{m, k}-a_{m, k-3}-\left\lceil b_{m, k-2} / 2\right\rceil+1$ lines of type $(0,1)$ of $Q$. The scheme $Y \cup E \cup F \cup v$ is a flat limit of a family of disjoint unions of $a_{m, k-1}+1$ lines, none of them containing $P$. By the semicontinuity theorem for cohomology to prove the existence of $B$ it is sufficient to prove that $h^{0}\left(\mathcal{I}_{m P \cup Y \cup E \cup F \cup v}(k-1)\right)=0$. Since $\operatorname{Res}_{Q}(m P \cup Y \cup F \cup E \cup F \cup v)=m P \cup Y \cup E \cup v$ and $Q \cap(m P \cup Y \cup F \cup E \cup F \cup v)=$ $(Y \cap Q) \cup E \cup F$, it is sufficient to prove that $h^{0}\left(Q, \mathcal{I}_{F \cup E \cup(Y \cap Q)}(k-1)\right)=0$, i.e. that $h^{0}\left(Q, \mathcal{I}_{(Y \cap Q) \backslash S}\left(k-1, k-a_{m, k-1}+a_{m, k-3}-2\right)\right)=0$. Since $(Y \cap Q) \backslash S$ is general in 
$Q$, it is sufficient to prove that $\sharp(Y \cap Q)-\sharp(S) \geq k\left(k-a_{m, k-1}+a_{m, k-3}-1\right)$. By (2) for the integer $k^{\prime}:=k-1$ we have $\sharp(Y \cap Q)-\sharp(S)=k\left(k-a_{m, k-1}+a_{m, k-3}-\right.$ $1)+k-b_{m, k-1}>k\left(k-a_{m, k-1}+a_{m, k-3}-1\right)$.

(b2) Now assume $k=m+4$. We modify the proof of $H_{m, m+3}$ (Lemma 5). We have $a_{m, m+1}=m+2, b_{m, m+1}=0, a_{m, m+3}=2 m+4$ and $b_{m, m+3}=4$ (Remark 1). Let $Y \subset \mathbb{P}^{3}$ be a general union of $m+2$ lines. By [4, Part (i)(c) of Theorem 4.2] we have $h^{i}\left(\mathcal{I}_{Y}(m+1)\right)=0, i=0,1$. For a general $Y$ we may assume that $Y \cap Q$ is formed by $2 m+4$ general points of $Q$. Let $F \subset Q$ be a general union of $m+3$ lines of type $(0,1)$. Use $Y \cup F$. Since $Y \cap Q$ is a general subset of $Q$ with cardinality $2 m+4$, we have $h^{0}\left(Q, \mathcal{I}_{Q \cap Y}(m+3,0)\right)=0$. We also have $Y \cap F=\emptyset$ and hence $h^{0}\left(Q, \mathcal{I}_{(Y \cap Q) \cup F}(m+3)\right)=0$.

\section{REFERENCES}

[1] T. Aladpoosh and E. Ballico, Postulation of disjoint unions of lines and a multiple point, Rend. Sem. Mat. Univ. Pol. Torino (to appear).

[2] E. Carlini, M. V. Catalisano and A. V. Geramita, Bipolynomial Hilbert functions, J. Algebra 324 (2010), no. 4, 758-781.

[3] E. Carlini, M. V. Catalisano and A. V. Geramita, 3-dimensional sundials, Cent. Eur. J. Math. 9 (2011), no. 5, 949-971.

[4] E. Carlini, M. V. Catalisano and A. V. Geramita, Reduced and non-reduced linear spaces: lines and points, arXiv: 1308.6796.

[5] R. Hartshorne, Algebraic Geometry, Springer-Verlag, Berlin-Heidelberg-New York, 1977.

[6] R. Hartshorne and A. Hirschowitz, Droites en position générale dans $\mathbb{P}^{n}$, in: Algebraic Geometry, Proceedings, La Rábida 1981, 169-188, Lect. Notes in Math. 961, Springer, Berlin, 1982.

[7] A. Hirschowitz, Sur la postulation générique des courbes rationnelles, Acta Math. 146 (1981), 209-230.

Dept. of Mathematics, University of Trento, 38123 Povo (TN), Italy

E-mail address: ballico@science.unitn.it 